\title{
The Effect of Microstructure on Stress-Induced Martensitic Transformation under Cyclic Loading in the SMA Nickel-Titanium
}

\author{
Michael Kimiecik ${ }^{\mathrm{a}}$, J. Wayne Jones ${ }^{\mathrm{a}}$, Samantha Daly $\mathrm{y}^{\mathrm{a}, \mathrm{b}, 1}$ \\ ${ }^{a}$ Department of Materials Science and Engineering, University of Michigan, 2300 Hayward, Ann Arbor, MI \\ 48109, USA \\ ${ }^{\mathrm{b}}$ Department of Mechanical Engineering, University of Michigan, 2350 Hayward, Ann Arbor, MI 48109, USA
}

\begin{abstract}
A combined experimental and analytical study to determine the configurations of transforming martensite during ambient temperature cyclic deformation of superelastic Nickel-Titanium has been conducted. Full-field, sub-grain-size microscale strain measurements were made in situ during cycling using distortion-corrected Digital Image Correlation combined with Scanning Electron Microscopy (SEM-DIC). Using grain orientation maps from Electron Backscatter Diffraction analysis, possible configurations of martensite formed during cyclic deformation were identified by matching the calculated and measured strain fields. This analysis showed that the inclusion of Correspondence Variants (CVs) in addition to Habit Plane Variants (HPVs) of transformed martensite was necessary to provide a robust fit between calculated and measured strain fields. The approach also provided evidence that there was a more rapid accumulation of residual strain in $\mathrm{CV}$ regions and that a correlation existed between residual strain accumulation and the loss of actively transforming martensite in later cycles. It was also found that regions of CVs could coexist with untransformed austenite and Habit Plane Variants (HPVs) in individual grains throughout the microstructure, and that these regions of CVs formed before the end of the macroscopic stress plateau. The CV structure that forms during the initial superelastic deformation of Nickel-Titanium plays a critical role in shaping and stabilizing subsequent martensite recovery during cyclic loading.
\end{abstract}

\section{Background}

Shape memory alloys (SMAs) are a class of metallic materials that can recover large strains through a thermally-controlled or stress-controlled phase transformation. When recovery occurs via a temperature change it is referred to as the shape memory effect, whereas superelasticity refers to recovery that occurs by the removal of load (Bhattacharya, 2003; Miyazaki et al., 1983). The shape memory and superelastic effects are caused by a solid-to-solid phase transformation

\footnotetext{
${ }^{1}$ Corresponding Author: Department of Mechanical Engineering, Department of Materials Science and Engineering, University of Michigan, 2350 Hayward, Ann Arbor, MI 48109-2125, USA, e-mail: samdaly@umich.edu , phone: 1-734-763-8137
}

(C) 2016. This manuscript version is made available under the Elsevier user license http://www.elsevier.com/open-access/userlicense/1.0/ 
between a high-symmetry austenite phase and one of several martensite variants, although each effect is produced by transformation along different thermomechanical routes. In the case of Nickel Titanium, austenite has a B2 cubic structure and the martensite variants are B19' monoclinic (Bhattacharya and Kohn, 1996; Bhattacharya, 2003; Hane and Shield, 1999; Matsumoto et al., 1987; Miyazaki et al., 2000). The transition between these two phases can produce up to $10 \%$ strain along certain crystallographic directions. Typically, transformation and recovery occur at constant stress which causes a flag-shaped hysteresis loop in the macroscopic stress-strain curve of superelastic Nickel Titanium, as shown in Figure 1. Superelastic transformation is considered to be complete at the end of the upper stress plateau of these curves.

[Figure 1 placed approximately here]

Under displacement-controlled cyclic loading, superelastic compositions of Nickel Titanium exhibit functional fatigue (Eggeler et al., 2004; Kang, 2013; Kang et al., 2009; Kim and Daly, 2010; Kumar et al., 2011; Norfleet et al., 2009; Pelton, 2011; Richards et al., 2013; Yamamoto et al., 2013). Functional fatigue is defined by a shift in the characteristic strains and stresses associated with martensitic transformation and recovery during cycling, as shown in Figure 1. In superelastic material, the stress required to nucleate a macroscopic martensite band tends to decrease with cycling, along with the total recovered transformation strain, resulting in a reduction in total hysteresis over each subsequent cycle. Macroscopically, superelastic samples accumulate residual strain under cycling, which is a mixture of locked-in martensite and plasticity (Kang, 2013; Kang et al., 2009; Richards et al., 2013). The macroscopic changes that occur after cycling a specimen from macroscopic austenite to macroscopic martensite and back can also be observed during incomplete cycling of the material, for example during the pulsile loading of SMA cardiac stents (Pelton, 2011; Pelton et al., 2008; Robertson et al., 2011; Shaw, 1997). Each section of the macroscopic stress-strain response is independently affected by loading history, indicating that local history impacts later martensite transformation. If these history effects were a global phenomenon, partial transformation cycles would affect the entire stress-strain response rather than producing segment-by-segment drops in the nucleation and propagation stress observed over previously traversed macroscopic strains. This piecewise sensitivity to loading history is indicative of structural changes taking place in areas of the SMA after each portion independently transforms between austenite and martensite. The details of this structural change, whether it is related to a change in how the martensite forms or due to residual damage associated with martensite transformation, is the subject of much previous research and is a motivator of the work presented here.

Recent experimental and computational work indicates that plasticity occurs concurrently with martensitic transformation, rather than as a subsequent process at the end of the macroscopic stress plateau. Neutron diffraction studies have been used to differentiate elastic, transformation, 
and plastic strains in polycrystalline specimens. In the early stages of loading, elastic strain in the austenite phase stabilizes at approximately 0.0025 axial strain prior to the start of martensitic transformation (Stebner et al., 2013). However, as loading continues, plastic deformation plays an active role prior to the saturation of initial macroscopic martensitic transformation Measurements indicate that strain induced rehardening of transformed material starts in superelastic tension when the sample reaches nominally $75 \%$ of the strain necessary to macroscopically exhaust the initial transformation to martensite in the specimen. Additionally, a recent micromechanical model of superelastic transformation in a generalized 2-D shape memory alloy includes plasticity as a possible deformation mechanism and demonstrates how the simultaneous activity of slip systems can bridge incompatibilities between regions of martensitic transformation in a polycrystalline shape memory alloy (Richards et al., 2013).

One of the difficulties in considering that plasticity occurs concurrently with martensitic transformation is that compatibility between the austenite and martensite variants affects the development of dislocation networks, and thus the character of functional fatigue. The intensification of dislocation networks across superelastic cycles in a typical superelastic alloy, where there is no compatibility between the austenite and lattice correspondence variants of martensite, is a possible explanation of the strain memory effect (Kim and Daly, 2013, 2010) and return point memory (DesRoches et al., 2004; Ortin et al., 2006; Rao et al., 2014). Here, the strain memory effect refers to a return to the mesoscale/microscale transformation configuration with cycling, and return point memory refers to the similarity in macroscopic stress-strain curves with incomplete cycling. These effects are the meso- and macroscopic consequences, respectively, of the repeated transformation of austenite to a particular set of martensite variants at the microstructural length scale. This set of variants is stabilized or 'blueprinted' by persistent dislocation networks formed at the boundaries of martensite laths during the first superelastic cycle.

In materials without austenite-martensite compatibility, dislocation networks that form during initial martensitic transformation have been observed to persist for multiple superelastic cycles. Using in situ mechanical testing in a transmission electron microscope, superelastic test coupons that were macroscopically transformed into martensite showed extensive dislocation networks in their unloaded state (Frick et al., 2010; Norfleet et al., 2009; Pelton, 2011; Pfetzing-Micklich et al., 2012). The importance of martensitic transformation towards the formation of dislocation networks was further highlighted by combined experiments and modeling of superelastic single crystal micropillars, in which dislocation loops formed as the austenite was 'pinched' between two martensite variants that constituted a growing twin. Drawn out dislocation loops appeared solely where martensite twins formed, based on the boundary of the looped and dislocation-free austenite pillar (Bowers et al., 2014; Norfleet et al., 2009; Pfetzing-Micklich et al., 2012). These 
findings indicate that dislocation structures can cross the austenite-martensite phase boundary during cyclic loading, and that these mobile dislocations accumulate with each loading cycle. Similarly, pulsile fatigue experiments of superelastic material produced bands of plastically deformed austenite, and areas of retained martensite that exhibited lower dislocation density as determined by TEM (Pelton, 2011). Dislocations formed at the martensite-austenite interface and in the wake of martensite recovery, but appeared to be located principally in the austenite phase. These dislocation networks are intimately related to martensite/austenite compatibility, as they are unobserved in alloys where the two phases maintain coherent boundaries. In materials where compatibility exists between the austenite phase and each individual martensite variant (referred to as $\lambda_{2}=1$ alloys, in reference to the middle eigenvalue of their martensite Bain matrices), both the accumulation of residual plastic strain and the persistence of martensite structures with cycling are small (Song et al., 2013).

As evident in the micropillar experiments, the compatibility conditions between martensite and austenite during transformation lead to the close association of dislocations with martensiteaustenite interfaces. Though the interface between twinned martensite and austenite is commonly referred to as a habit plane, implying compatibility between the two phases, the crystallographic compatibility of the B2 to B19' transformation is not perfect for near equiatomic Nickel Titanium (Madangopal, 1997; Miyazaki et al., 1989a, 1989b, 1984). Refinement of the twinned martensite laths and a zone of elastically deformed material is needed to maintain compatibility across the atomically sharp austenite-martensite interface. The component correspondence variants (CVs) that combine to create a habit plane variant (HPV) are not individually compatible with the austenite, and thus cannot be accommodated with an elastic boundary layer. Modeling work (Bowers et al., 2014) has attributed the formation of residual dislocation loops in the micropillar experiments to the addition of a compatibility stress at the martensite/austenite interface to the global applied stress. In other alloys exhibiting perfect compatibility between the austenite and martensite phases, this additional elastic zone does not exist and dislocation networks do not appear to form (Chen et al., 2013; Delville et al., 2010; Shi et al., 2014; Song et al., 2013). When thermally cycled, these alloys exhibited no strain memory during the shape memory process; different martensite variants formed in different patterns with each transformation from austenite to martensite. The lack of strain memory in fully compatible alloys strongly suggests that dislocation networks resulting from martensite-austenite incompatibility have a critical role in the secondary formation of martensite in cyclic applications. Without the dislocation network that results from incompatible correspondence variants to provide a stable blueprint guiding martensitic transformation, the pattern of martensite variants in subsequent cycles becomes random (Song et al., 2013). 
Direct evidence of the coordination between correspondence variants, residual strain, and strain memory has not been shown in alloys that exhibit strain memory behavior. This work captures, in situ, the interactions between plasticity and martensitic transformation in a polycrystalline material by using an approach that allows for the examination of their local configuration and coexistence. Here, austenite-martensite phase compatibility is linked to residual strain accumulation in a polycrystal. It was found that areas that principally transform to an incompatible combination of austenite and martensite accumulate significantly more residual strain than compatible configurations. Additionally, these results indicate that there is minimal penalty in forming tortuous interfaces between austenite and multiple habit plane variants of martensite, if all present phases are compatible. Depending on the assumptions used in tracking transformation, the residual strain networks can be attributed to either a plastic zone that favors habit plane variant formation or the retention of large portions of the initially transformed martensite. These findings result from the use of novel full-field microscopy techniques to experimentally investigate the interplay between martensitic transformation and plasticity in a polycrystal.

\section{Material and Experimental Setup}

Superelastic dogbone-shaped specimens were machined from SE508 sheet obtained from Nitinol Devices and Components, Inc. using electrical discharge machining. Samples were ground with 320 grit metallographic paper to remove the surface oxide. Following the initial grinding, samples were recrystallized in air at $625^{\circ} \mathrm{C}$ for 10 minutes, followed by 10 minutes at $450^{\circ} \mathrm{C}$. This resulted in a mean grain diameter of $3.65 \mu \mathrm{m}$ as measured by electron backscatter diffraction (EBSD). Samples were then mechanically polished from 320 to 800 grit silicon carbide paper, followed by diamond suspensions from $6 \mu \mathrm{m}$ to $1 \mu \mathrm{m}$, with the final step being vibratory polishing with colloidal silica to produce a mirror finish. Platinum fiducial markers were applied to samples to align the strain fields with crystallographic information gathered from EBSD. The EBSD scan performed after laying these fiducial markers was taken over a $100 \times 100$ $\mu \mathrm{m}$ area with a $200 \mathrm{~nm}$ step size. The EBSD map was then resampled using nearest neighbors for point-by-point alignment with the strain maps. Samples were patterned for Digital Image Correlation (DIC) using self-assembled gold nanoparticles deposited on a silane monolayer (Kammers and Daly, 2013a).

Surface displacements were measured using distortion-corrected Digital Image Correlation paired with Scanning Electron Microscopy (SEM-DIC) while the sample was loaded in situ using a screw-driven Kammrath and Weiss SEM stage (Figure 2). DIC is a non-contact optical technique developed in the 1980s for use with CCD cameras (Schreier et al., 2009) and has since been adapted for use with SEM imaging (Sutton et al., 2007a, 2007b, Kammers and Daly, 2013b). After correcting for the spatial and drift distortions that are unique to SEM imaging, DIC 
can detect displacements as small as 0.02 pixels (Reu, 2013; Wang et al., 2011). Local strains can be calculated from the resulting high resolution, high precision displacement maps. The images used to generate the strain maps in this study had a spatial resolution of $15 \mathrm{~nm} / \mathrm{pixel}$. Strain was calculated from these displacements over a 9 × 9 pixel window surrounding each interrogation point and a step size of three pixels in each direction, which resulted in a typical 4 $\mu \mathrm{m}$ diameter grain having nominally 6000 strain points available for analysis.

An advantage of SEM-DIC is the ability to independently analyze a large number of sub-grain data points over multiple deformation cycles. This enabled the characterization of martensitic transformation in situ over multiple loading cycles at high resolution. Since all calculated displacements, and subsequently all strain measurements, were made against a single reference image, measurements of the same material point could be compared over its loading history. Thus the deformation of a single grain, or subgrain region, could be compared between any number of loading cycles in reference to the local characteristics of the material, including topographical character, chemical composition, and phase information. The grain orientation was aligned with strain data to enable predictions of transformation and its evolution, and to compare these predictions point-by-point against the experimentally-measured strain data. This enabled a large number of point-by-point samples of many crystal orientations to be fit to theoretical predictions of their transformation behavior. Because of the highly heterogeneous nature of this transformation on the microscale, this sub-grain point by point tracking and full field approach across large numbers of grains was necessary to determine the patterns of transformation behavior. The analysis of the local crystal orientation and strain data to identify phase information is detailed in the following section.

\section{Methods}

A limitation of the strain-based analysis of materials is the inability to attribute strain to specific deformation mechanisms without additional information at each measurement point. Previous work by the authors at the microstructural length scale experimentally measured traces of localized strain and related these to the analytically-derived traces of crystallographic planes for possible transformation variants or slip systems (Kammers and Daly, 2013b; Kimiecik et al., 2013). This process was limited to identifying isolated martensite laths or clearly defined slip traces in austenite. Here, the configuration of habit plane variants and correspondence variants that best matches the experimentally measured surface strain is calculated on a point-by-point basis across large fields of view. The procedure to achieve this is described below.

\section{Determining Reference Strains for Each Possible Martensite Variant}

Experimentally measured strains were correlated to theoretically calculated transformation strains by first calculating the transformation strains of all possible correspondence variants 
(CVs) and habit plane variants (HPVs). To accomplish this, the Bain Transformation Matrices $\left(\boldsymbol{U}_{\boldsymbol{i}}\right)$ were calculated for each of the 12 martensite $\mathrm{CVs}$ possible from the undeformed austenite configuration based on the known lattice parameters for the martensite phase (Bhattacharya and Kohn, 1997; Bhattacharya, 2003; Hane and Shield, 1999; Knowles and Smith, 1980; Matsumoto et al., 1987). For Nickel Titanium, these have the form of $\boldsymbol{R}^{\boldsymbol{T}} \boldsymbol{U}_{\mathbf{1}} \boldsymbol{R}$ where $\boldsymbol{R}$ is a member of the cubic symmetry group $\mathcal{L}_{\text {cubic }}$ and $\boldsymbol{U}_{\mathbf{1}}$ has the form:

$$
\boldsymbol{U}_{1}=\left[\begin{array}{lll}
\gamma & \epsilon & \epsilon \\
\epsilon & \alpha & \delta \\
\epsilon & \delta & \alpha
\end{array}\right]
$$

The following parameters were used: $\gamma=0.9563, \epsilon=-0.0427, \alpha=1.0243$, and $\delta=0.058$ (Hane and Shield, 1999). From the Bain stretch matrices, transformation strains for each of the correspondence variants were determined from continuum mechanics. To simplify the analysis and have the proper work conjugate to the experimentally measured engineering stresses, the Biot strain was used. The Biot $\left(\boldsymbol{\varepsilon}_{\boldsymbol{B i o t}}\right)$ strain is defined as

$$
\varepsilon_{\text {Biot }}=\boldsymbol{U}-\boldsymbol{I}
$$

where $\boldsymbol{I}$ is the identity matrix.

After calculating the Biot transformation strains $\left(\varepsilon_{C V}^{i}\right)$ and Bain matrices for the 12 correspondence variants, effective Bain matrices were constructed for each of the $192 \mathrm{HPVs}$ [please see (Ball and James, 1989; Bhattacharya, 2003; Gall and Sehitoglu, 1999; Hane and Shield, 1999) for details on this procedure], as well as the relative fractions of CVs in each HPV. The effective Bain matrix of an HPV is a linear combination of the Bain stretch matrices of its $\mathrm{CV}$ components, and represents the mean stretch that results from austenite transforming to the HPV combination. From the relative volume fractions of each CV in the HPV ( $\vartheta$ and $1-\vartheta)$, the Biot strain for each HPV in the reference configuration was given by:

$$
\vartheta \varepsilon_{C V}^{n 1}+(1-\vartheta) \varepsilon_{C V}^{n 2}=\varepsilon_{H P V}^{n}
$$

Note that the Biot strain is assumed to be linear with the Bain stretch matrix. The compatibility of each HPV with all other HPVs was then determined from the calculated strain of each HPV by a zero-sum shear definition (Gall and Sehitoglu, 1999). HPV pairs whose sum resulted in purely dilatational strain, i.e.

$$
\epsilon_{i j k} \epsilon_{p q r}\left(\varepsilon_{j q}^{n}-\varepsilon_{j q}^{m}\right)=0
$$

where $\epsilon_{i j k}$ is the permutation tensor, were defined as compatible. Other definitions of compatibility, such as the formal calculation of habit planes for each HPV pair, can be used to limit the potential solution set. The relatively tolerant compatibility condition of Equation 4 was 
chosen to allow for the maximum possible combinations of compatible HPVs (1650 for the 96 Type II HPVs) and for less-than-perfect compatibility between phases. However, it may overestimate the ability of HPVs to coexist. Independent of the compatibility definition used here, it was necessary to allow for multiple HPVs to exist at a given point because of the smeared nature of DIC strain calculation. Local strain can be effectively mixed with the immediate surroundings of an individual point, and thus a similar mixing needed to be allowed in the phase fitting process.

\section{Transforming Reference Strain to Local Grain Strain}

In order to match the experimental data to the calculated strain values, the reference strains for each $\mathrm{CV}$ and HPV were rotated into the same orientation as the grain under investigation. Using the Euler Angles collected from EBSD, a rotation matrix $(\boldsymbol{R})$ was constructed to transform matrices from the reference to the grain orientation in $\mathrm{X}-\mathrm{Y}-\mathrm{Z}$ space. Note that the inverse procedure cannot be accomplished due to the inability to experimentally measure out of plane strains. All the rotated strain values were stored in the new local configuration:

$$
\boldsymbol{\varepsilon}^{\prime}=\boldsymbol{R} \boldsymbol{\varepsilon} \boldsymbol{R}^{T}
$$

For each grain, a strain space representation of each HPV and CV was created based on the rotated $\left(\varepsilon_{x x}^{\prime}, \varepsilon_{y y}^{\prime}, \varepsilon_{x y}^{\prime}\right)$ strain values. These points will later be compared against experimental data to determine the most probable HPV and CV transformation combination.

\section{Matching Local Strain to Measured Strain}

Each measured displacement (strain) data point in a grain was independently matched to the HPV and CV solution cloud detailed in the last section. The $i$ th strain point $\left(\boldsymbol{\varepsilon}^{i}\right)$ was placed in the same strain space as all of the rotated HPV and CV representations $\left(\varepsilon_{H P V}^{n}\right.$ and $\left.\varepsilon_{C V}^{m}\right)$ and compared to several possible HPV and CV solutions. The mathematical constructions used to compare each test point to possible solutions of each grain were based on a distance minimization in strain space. In the case of two-phase mixtures (e.g. austenite with HPV), the distance from the test point to the line segment connecting each phase's point in strain space was minimized. Similarly, for three-phase mixtures (e.g. austenite with two compatible HPVs) the distance minimization was performed between the test point and the triangular plane connecting the three phase points. The appropriate phase fraction of martensite configurations and retained austenite in both cases was calculated using lever-law rules to find the combination with the lowest root mean squared error. These constructions are represented graphically in Figure 3 and mathematically below. 
The single HPV plus austenite solution was tested first. The minimum root mean squared error $\left(\Delta \varepsilon_{R M S}\right)$ from the $i^{\text {th }}$ strain point to a phase fraction $\left(\lambda_{H P V} \in(0,1]\right)$ of the nth $\operatorname{HPV}\left(\varepsilon_{H P V}^{n}\right)$ was determined by:

$$
\begin{gathered}
\Delta \varepsilon_{R M S}=\frac{\left|\lambda_{H P V}^{n} \varepsilon_{H P V}^{n}-\varepsilon^{i}\right|}{\sqrt{3}} \\
\lambda_{H P V}^{n}=\frac{\varepsilon_{H P V}^{n} \cdot \varepsilon^{i}}{\left|\varepsilon_{H P V}^{n}\right|^{2}}
\end{gathered}
$$

The $\Delta \varepsilon_{R M S}$ value is the baseline error for the strain point, where any other solution had to provide a lower error to be considered. After exhausting the single variant solutions, a similar process was used to determine the minimum RMS error for any pair of compatible HPVs (e.g., $\varepsilon_{H P V}^{n}$ and $\varepsilon_{H P V}^{m}$ that satisfy the conditions of equation 5) of any phase fraction combination,

$$
\begin{gathered}
\lambda_{H P V}^{n}, \lambda_{H P V}^{m} \in(0,1) \\
\lambda_{H P V}^{n}+\lambda_{H P V}^{m} \leq 1
\end{gathered}
$$

as follows:

$$
\begin{gathered}
\Delta \varepsilon_{R M S}=\frac{\left|\lambda_{H P V}^{n} \varepsilon_{H P V}^{n}+\lambda_{H P V}^{m} \varepsilon_{H P V}^{m}-\varepsilon^{i}\right|}{\sqrt{3}} \\
\varepsilon_{\text {in plane }}^{i}=\varepsilon^{i}-\left(\boldsymbol{\varepsilon}^{i} \cdot \frac{\left(\varepsilon_{H P V}^{n} \times \varepsilon_{H P V}^{m}\right)}{\left|\varepsilon_{H P V}^{n} \times \varepsilon_{H P V}^{m}\right|}\right)\left(\frac{\left(\varepsilon_{H P V}^{n} \times \varepsilon_{H P V}^{m}\right)}{\left|\varepsilon_{H P V}^{n} \times \varepsilon_{H P V}^{m}\right|}\right) \\
\boldsymbol{T}=\left[\begin{array}{ll}
\varepsilon_{H P V, 1}^{n} & \varepsilon_{H P V, 1}^{m} \\
\varepsilon_{H P V, 2}^{n} & \varepsilon_{H P V, 2}^{m} \\
\varepsilon_{H P V, 3}^{n} & \varepsilon_{H P V, 3}^{m}
\end{array}\right] \\
\left(\begin{array}{l}
\lambda_{H P V}^{n} \\
\lambda_{H P V}^{m}
\end{array}\right)=\boldsymbol{\varepsilon}_{\text {in plane }}^{i} / \boldsymbol{T}
\end{gathered}
$$

For points that transformed into more than 50\% HPV (as determined by the sum of $\lambda_{H P V}$ values of any single or multiple HPV-only variant solutions), the possibility of detwinning of the primary HPV $\left(\varepsilon_{H P V}^{n}\right)$ into its component CVs $\left(\varepsilon_{C V}^{n 1}, \varepsilon_{C V}^{n 2}\right)$ was checked. This was performed following the same mathematical framework as in the multiple HPV solution, where $\varepsilon_{H P V}^{n}, \varepsilon_{H P V}^{m}$, and their respective $\lambda_{H P V}$ values were replaced with $\varepsilon_{C V}^{n 1}, \varepsilon_{C V}^{n 2}$, and the corresponding $\lambda_{C V}$ :

$$
\begin{gathered}
\Delta \varepsilon_{R M S}=\frac{\left|\lambda_{C V}^{n 1} \varepsilon_{C V}^{n 1}+\lambda_{C V}^{n 2} \varepsilon_{C V}^{n 2}-\varepsilon^{i}\right|}{\sqrt{3}} \\
\varepsilon_{\text {in plane }}^{i}=\varepsilon^{i}-\left(\boldsymbol{\varepsilon}^{i} \cdot \frac{\left(\varepsilon_{C V}^{n 1} \times \varepsilon_{C V}^{n 2}\right)}{\left|\varepsilon_{C V}^{n 1} \times \varepsilon_{C V}^{2}\right|}\right)\left(\frac{\left(\varepsilon_{C V}^{n 1} \times \varepsilon_{C V}^{n 2}\right)}{\left|\varepsilon_{C V}^{n 1} \times \varepsilon_{C V}^{n 2}\right|}\right)
\end{gathered}
$$




$$
\begin{gathered}
\boldsymbol{T}=\left[\begin{array}{ll}
\varepsilon_{C V, 1}^{n 1} & \varepsilon_{C V, 1}^{n 2} \\
\varepsilon_{C V, 2}^{n 1} & \varepsilon_{C V, 2}^{n 2} \\
\varepsilon_{C V, 3}^{n 1} & \varepsilon_{C V, 3}^{n 2}
\end{array}\right] \\
\left(\begin{array}{l}
\lambda_{C V}^{n 1} \\
\lambda_{C V}^{n 2}
\end{array}\right)=\boldsymbol{T}^{-1} \varepsilon_{\text {in plane }}^{i}
\end{gathered}
$$

If the detwinned volume fractions were between zero and one, the twinned $\left(\varphi_{t}\right)$ and detwinned $\left(\varphi_{d t}\right)$ fractions were calculated based on the volume fraction $(\vartheta)$ of $\mathrm{CV}$ s in the twin from (Eq. 2:

$$
\begin{gathered}
\varphi_{t}=\left\{\begin{array}{c}
\frac{\lambda_{C V}^{n 1}}{\vartheta}, \text { if } \frac{\lambda_{C V}^{n 1}}{\vartheta} \leq \frac{\lambda_{C V}^{n 2}}{1-\vartheta} \\
\frac{\lambda_{C V}^{n 2}}{1-\vartheta}, \text { if } \frac{\lambda_{C V}^{n 1}}{\vartheta}>\frac{\lambda_{C V}^{n 2}}{1-\vartheta}
\end{array}\right. \\
\varphi_{d t}=\left\{\begin{array}{c}
\lambda_{C V}^{n 2}-\frac{\lambda_{C V}^{n 1}}{\vartheta}(1-\vartheta), \text { if } \frac{\lambda_{C V}^{n 1}}{\vartheta} \leq \frac{\lambda_{C V}^{n 2}}{1-\vartheta} \\
\lambda_{C V}^{n 1}-\frac{\lambda_{C V}^{n 2}}{1-\vartheta} \vartheta, \text { if } \frac{\lambda_{C V}^{n 1}}{\vartheta}>\frac{\lambda_{C V}^{n 2}}{1-\vartheta}
\end{array}\right.
\end{gathered}
$$

If the total volume fraction of HPVs in the initial solution was less than $50 \%$, there was no need to calculate $\lambda_{C V}^{n}$, and the following conditions were set:

$$
\begin{gathered}
\varphi_{t}=\sum_{n} \lambda_{H P V}^{n} \\
\varphi_{d t}=0
\end{gathered}
$$

The configuration with the lowest $\Delta \varepsilon_{R M S}$ was selected as the best fit for each point. All identifiers for the twinned and detwinned structures and their volume fractions were recorded for further analysis, such as correlating transformation fraction to residual strain as will be discussed later.

Considering HPV combinations in addition to their fundamental CV components may seem redundant, but it is necessary to examine both the enhanced compatibility of HPV structures with the parent austenite and with each other. The above algorithm could be recreated to only consider mixtures of CVs, as all the HPV structures would be a subset or special case of the complete CV solution set. The overall fit and error of such a $\mathrm{CV}$-only solution would be comparable to explicitly including the HPV sub-solutions. However, this would require the use of strict compatibility between $3+\mathrm{CV}$ solutions and would lose the flexibility of the HPV compatibility conditions used here, which are more suitable when working with experimentally-obtained strain fields. Also, it would require additional steps to determine the maximum compatibility of a CV mixture with the parent austenite if HPV solutions were not explicitly included at the outset. For 
these reasons, the redundancy of explicitly including the HPV solutions in the CV solution set was considered acceptable.

A shortcoming of this analysis is the lack of a guaranteed unique solution when only in-plane strains are measured, as is this case with surface strain measurement techniques like 2-D (or 3-D) DIC. Of the six independent strain components that describe each CV, HPV, or their combinations, only three could be matched to experimental data. Unique identification of the exact configuration of martensite was therefore not guaranteed, as symmetry related variants can be indistinguishable in three-dimensional strain space and multiple HPVs can occupy identical $\varepsilon_{H P V}$ points. At maximum, four distinct variant strain points in 6-D strain space can share the same 3-D in-plane projection. However, there is sufficient information to determine the configuration types present in a local mixture. For example, it is possible to determine if a point lies on a line connecting several coincident, but incompatible, HPVs to the origin; when this happens, it is strong evidence that the local strain is a result of a single HPV, though determining which specific HPV may not be possible. Similarly, it is possible to determine when a point lies outside the bounds of all HPV solutions, which requires the presence of detwinned CVs. The relative position outside the HPV bound can also assist in the determination of the direction in and degree to which the detwinning process progressed, given the sparse distribution of the 12 possible CV solutions compared to the 192 HPVs and the lack of duplicity in the CV solutions. Thus, the best fit determined by this method may be characterized as a set of non-unique solutions which all fit the experimental data equally well. However, the properties of the members of the solution set, such as their detwinned and twinned volume fractions, can be defined through strain analysis alone.

\section{History Dependence}

For multi-cycle analysis, the solution set of martensite variants can be limited based on transformation history. The algorithm has no preference to repeat the same configuration of martensite in consecutive cycles; the solution with the lowest error will be found independently for each cycle if additional constraints are not applied. This corresponds to the assumption that martensite is infinitely reorientable during each transformation cycle and that transformation has no history bias, which we will term as the history-free condition. As previous work (DesRoches et al., 2004; Kim and Daly, 2010, 2013; Ortin et al., 2006; Rao et al., 2014) has shown a strong strain memory effect, where the same martensitic configuration repeatedly transforms and recovers, a strict history dependent addition was made to the code to restrict transformation under cycling. With the strict history assumption, all possible martensite variants are made available in the first cycle, and all subsequent fitting is done only with variants that appeared in the first loading cycle. Each point is restricted independent of its neighbors. This conforms with strain memory behavior observed on the macro and micro length scales, as it is likely from the 
high degree of similarity in the strain data for each cycle at maximum load that martensite transformation is repeating in each cycle. Both the history-free and strict history conditions are calculated in this paper to compare the two extremes of history dependence against experimental data.

\section{$\underline{\text { IV. Results and Discussion }}$}

The use of distortion-corrected SEM-DIC coupled with the analysis described in Section III led to a number of findings, including the determination that Correspondence Variants (CVs) formed during initial martensitic transformation, that there was a more rapid accumulation of residual strain in those $\mathrm{CV}$ regions, and that there exists a correlation between residual strain accumulation and the loss of actively transforming martensite in later cycles. It was also found that regions of CVs could coexist with untransformed austenite and Habit Plane Variants (HPVs) in individual grains throughout the microstructure, and that these regions of CVs formed before the end of the macroscopic stress plateau. These and other findings are discussed in detail below.

First, it was determined that regions of CVs coexisted with regions of untransformed austenite and HPVs in individual grains throughout the microstructure. Additionally, these CV regions formed in the microstructure early in the superelastic cycle, well before the end of the macroscopic stress plateau. Prior experimental work (Stebner et al., 2013) suggests that significant reorientation of martensite variants does not occur until later in the superelastic deformation process, when the stress begins to rise above the transformation plateau; interestingly, it has generally been assumed that CVs do not form before this reorientation process due to their incompatibility with the parent austenite. However, if the theoretical analysis was restricted to single and multiple HPV solutions (Figure 4b), significant differences with the experimental data arose (Figure 4a). Specifically, HPV solutions could be found for data points with $\leq 0.06$ axial strain, but failed to account for regions that reached upwards of 0.1 axial strain. A strong match between the theoretically calculated strains and the experimentally measured strains could only be achieved by allowing detwinning in addition to the HPV solutions (Figure $4 c)$.

Large amounts of material transformed to $\mathrm{CVs}$ and recovered to austenite during the first cycle, as shown in Figure 5. Correspondingly, the first superelastic cycle showed the greatest recoverable strain, both in the macroscopic stress-strain response and in the grain-level SEMDIC strain fields. As cycling continued, the volume fraction of recoverable CV decreased in most areas - rapidly in the early deformation cycles, and later stabilizing - giving way to an increase in HPV volume fraction. The nominally five or six cycles needed to stabilize the CV fraction (Figure 5) corresponded to the time taken to stabilize both the global and local responses, including the rate of shakedown in the macroscopic stress-strain response (Figure 6a) 
and the rate of residual strain accumulation in the local microstructure (see Inset F in Figure 1). When the strict history assumption was used, $81 \%$ of the retained strain was attributed to the decrease in $\mathrm{CV}$ fraction. The remaining locked-in strain was likely due to plastic deformation, as the recoverable strain from HPVs increased during the same period. In contrast, if the historyfree assumption was used, the residual strain exhibited no dependence on the loss of martensite in the $\mathrm{CV}$-attributed points; across all strains attributed to a CV configuration, the residual strain remained a constant 0.03 axial strain. In this case, much of the loss in $\mathrm{CV}$ volume fraction was attributed to the reorientation of CVs into HPV configurations. The reasons for the discrepancy between the strict history and history-free analyses are addressed below.

Areas that initially transformed with a higher $\mathrm{CV}$ volume fraction tended to accumulate greater amounts of strain in subsequent cycles (Figure 6d). A plot of residual strain accumulation over ten cycles as a function of the $\mathrm{CV}$ fraction in cycle 1 is shown in Figure 7 . In this plot, the residual strains taken at all data points that transformed with the same $\mathrm{CV}$ fraction in cycle 1 were averaged and then tracked from cycle 1 through cycle 10. Certain CV fractions had significantly more data points to average than others; the majority of points were clustered around 0 and $1 \mathrm{CV}$ fraction, but no decade (range of 0.1 width) of $\mathrm{CV}$ fraction contained less than $1 \%$ of the total points. In Figure 7, there is a trend of higher CV volume fraction in Cycle 1 leading to greater residual strain in later cycles. The trend is nominally linear with $\mathrm{CV}$ fraction and saturated in later cycles. The greater accumulation of residual strain in regions with higher $\mathrm{CV}$ volume fraction can be explained by the lack of compatibility between austenite and single CVs. While HPVs can maintain an interface between austenite and transformed martensite, no such interface can be maintained between a pure CV and austenite. Large elastic zones (such as those bridging austenite and martensite in the HPV structure) or plasticity are likely candidates for maintaining compatibility between these incompatible phases. Given that initial transformation is largely recoverable, with significant residual strain only appearing in later cycles, there is likely an evolving combination of mechanisms active in maintaining the compatibility of the CV phases. Previous TEM work (Bowers et al., 2014; Norfleet et al., 2009; Pelton, 2011) examining the multiplication of dislocation structures in superelastic SMAs indicates that whatever small amount of plasticity was required to maintain initial compatibility of transformation likely grew and eclipsed other deformation mechanisms. The fact that only $80 \%$ of the local residual strains could be accounted for by a decrease in martensite volume fraction also supports an increase in plastic strain with cycling.

The linear relationship between $\mathrm{CV}$ fraction and residual strain accumulation indicates a continuous breakdown of the habit plane compatibility between the austenite and martensite phases. As a HPV moves away from the ratio of component $\mathrm{CV}$ s toward a pure $\mathrm{CV}$ configuration, there is a continuous increase in the additional plastic deformation required to 
maintain compatibility with the austenite phase. This is due to the finite strain incompatibility between austenite and CVs and the effective zero strain incompatibility between austenite and HPVs. An increasing fraction of $\mathrm{CV}$ that moves away from the HPV combination incurs a linearly increasing incompatibility strain (according to Equation 3) until the sample is 100\% CV. The experimental observation of a linear relationship between residual strain and initial fraction of $\mathrm{CV}$ in the recoverable transformation supports the rule of mixtures approach taken in the analysis.

The accumulation of residual strain in the polycrystal was not uniform, wherein areas of high recovered strain in the first cycle (indicating large amounts of reversible martensitic transformation) were the same areas that later showed high residual strain at the end of cycling. A possible explanation for this is that $\mathrm{CV}$ formation and recovery is not as favorable a mechanism as HPV formation and recovery. The incompatibility of CVs with the parent martensite requires a finite amount of strain to be accommodated by other means at their interface, presumably by plastic deformation. While this initial additional strain is small and does not impede reverse transformation, as seen by the initially high recoverable strain in the first superelastic cycle, it inevitably builds and compounds in the material. HPVs, being inherently compatible with the parent phase have no need for the plastic bridges of the CV-Austenite interface and are thus not inhibited from recovering in later cycles. This distinction between CV and HPV recovery becomes most apparent when comparing multiple HPV and CV/HPV transformation and recovery.

Linear combinations of HPVs, which lie on the same planes in strain space as CV/HPV combinations, showed no elevated residual strain accumulation compared to single HPV solutions. Considering the rule of mixtures approach for the mixing of two compatible HPVs, the low residual strain is the result of two zero strain endpoints along the mixture line. Any composition that can be created from the two compatible HPVs can maintain a compatible interface with the parent austenite, though it may be serrated depending on the HPV geometry. The recovery of CVs is impeded with increased cycling. However, martensite configurations that included only HPVs showed a greater propensity to recover over multiple cycles. Previously, it was shown that points that transformed to $\mathrm{CV}$ configurations accumulated greater residual strain with cycling. These same points also showed large drops in the total volume fraction of martensite lost between the first and last superelastic cycles. Even points that deviated from a pure HPV configuration by a few percent showed a large martensite fraction loss compared to HPV-only configurations. This indicates a minimal penalty for a tortuous habit plane that flips between multiple orientations in an HPV combination, as long as the individual boundary segments maintain compatibility with the parent phase. 
The saturation of residual strain and stabilization of CV fraction that occurs with cycling corresponds to macroscopic shakedown and leads to a more uniform distribution of HPVs. The path of this transformation evolution can be traced across multiple loading cycles by tracking sets of points that transform to various volume fractions of HPV, CV, and austenite. By averaging the volume fraction change of many points with the same initial composition of transforming HPVs and CVs, trends in how the transforming volume fractions shift with cycling can become more apparent. The gathered data was mapped onto an evenly distributed triangular mesh of representative phase fraction points through the Austenite-HPV-CV phase space based on the transformation volume fractions of the experiment's first cycle (Figure 6bc, Cycle 1). Points were assigned to the nearest mesh point, and the average displacement of all points assigned to each mesh point defined the mesh displacement. By cycle two these initially evenly distributed mesh points collapsed into a primarily HPV configuration. This process reached a stable configuration by cycle five or six, corresponding with the shakedown of the macroscopic stress-strain curve and the saturation of the residual strain (Figure 6ad). Thus, shakedown can be attributed in part to a shift in martensite volume fractions from configurations that are incompatible with the parent austenite to compatible configurations that are stabilized by plastic deformation.

The location of stable (shaken down) martensite depended on the amount of reorientation present in the local microstructure. If the history-free assumption was used (Figure 6b), which is analogous to an infinitely reorientable martensite configuration, all points collapsed to a dense, primarily HPV configuration with a high transforming volume fraction of martensite. While the loss of recoverable strain in this configuration matched well with experimental results, it required unreasonable assumptions about the residual strain field, discussed shortly. Alternatively, one can limit transformation to the martensite variants present in cycle 1 (Figure 6c), which represents the strict history assumption. This produced a more homogenous transformation, where every point containing some correspondence variant fraction collapsed to a low-martensite fraction, primarily HPV configuration. Points with no CV component, along the Austenite-HPV edge in Figure 6c, maintained their pure HPV character and slightly higher volume fractions of martensite. The true configuration of martensite in the shaken down superelastic sample likely lies between these two extremes, but as the methods presented here can only give $a$ best-fit configuration as opposed to the best-fit configuration, the results are conditional on which phases were included in the fit.

Similarly, the degree of reorientability of martensite during cyclic superelastic transformation informed the source of residual strain. Depending on the use of either the history-free or stricthistory assumption, less than $20 \%$ of the residual strain was due to sources of plasticity other than locked-in martensite. Analysis using the history-free assumption of infinitely reorientable 
martensite resulted in a stable, shaken-down configuration with a high volume fraction of transforming martensite. Using this assumption, significant recoverable martensite volume fraction was maintained throughout the specimen with cycling, and the maximum loss of martensite was approximately 10\% volume fraction over ten cycles (Figure 6b). This 10\% maximum loss was nominally uniform across all configurations of HPV and CV martensite. In comparison, the strict-history case - using the assumption of no martensite reorientation (Figure $6 \mathrm{c}$ ) - resulted in the loss of approximately $50 \%$ of the martensite associated with non-HPV solutions and $30 \%$ of pure HPV configurations. When the decrease in strain associated with the loss of CV-bound martensite was considered in the history-free case, it was weakly correlated with measured residual strains. The weak correlation between loss of martensite and residual strain indicates that if reorientation is prevalent during superelastic cycling, locked in martensite does not significantly contribute to residual strain beyond a small flat amount. Points with a non-reoriented CV loss had, on average, approximately a 0.03 residual axial strain, regardless of the total lost martensitic transformation strain (Figure 8a). The history-dependent loss in the CV strain, however, accounted for $80 \%$ or more of the residual strain on a point-by-point basis (Figure 8b). In either case, the residual strain included locked-in martensite, which was compatible with the martensite of the first cycle and promoted re-transformation to that same configuration.

A determination of how much residual strain is martensite and how much reorientation is present during superelastic cycling requires the further development of experimental capabilities. Diffraction-based analysis, which typically cannot measure local configurations over a wide area, could potentially be paired with the approaches used here to determine shifts in the martensite phase mixture. Tracking reorientation using diffraction-based methods would provide an additional input into the best fit model and allow the determination of the location of the shaken-down martensite configuration between the infinitely reorientable and strictly history dependent solutions. The residual strains measured in this work could then be ascribed to retained martensite or plasticity.

\section{$\underline{\text { V. Conclusions }}$}

A combined experimental and analytical approach was used to determine the configuration of transforming martensite during superelastic fatigue tests. Using distortion-corrected digital image correlation in a scanning electron microscope combined with theoretical analysis of martensite transformation strain based on orientation information from electron backscatter diffraction data, several findings were determined as follows:

- In order to fit experimental measurements, the inclusion of lattice correspondence variants $(\mathrm{CVs})$ in the solution set was required, even in the early stages of loading in the superelastic 
plateau. Regions of CVs coexisted with regions of untransformed austenite and HPVs in individual grains throughout the microstructure. Including CV solutions in addition to HPV solutions resulted in a strong match to the experimentally-measured strain fields.

- Large amounts of material transformed to CVs and recovered to austenite during the first cycle. Correspondingly, the first superelastic cycle showed the greatest recoverable strain, both in the macroscopic stress-strain response and in the grain-level SEM-DIC strain fields. As cycling continued, the volume fraction of recoverable $\mathrm{CV}$ decreased in most areas rapidly in the early deformation cycles- giving way to an increase in HPV volume fraction.

- Residual strain accumulated rapidly in areas that transformed into CVs in the first cycle, which can be explained by the lack of compatibility between austenite and single CVs. A linear relationship between initial CV fraction and residual strain accumulation indicates a continuous breakdown of the habit plane compatibility between the austenite and martensite phases.

- There was a strong correlation between residual strain accumulation at the microstructural length scale and macroscopic shakedown. When residual strain accumulation slowed in the microstructure, macroscopic shakedown stabilized.

- There were two possible paths for residual strain accumulation, depending on assumptions made about martensitic reorientation. With no penalty for reorientation, termed here as the history-free condition, only a small fraction of residual strain was attributed to retained martensite. Enforcing a repeated transformation path as observed in prior work, termed here as the strict history condition, led to the attribution of a large fraction of residual strain to locked-in martensite.

- The accumulation of residual strain in the polycrystal was not uniform. Areas of high recovered strain in the first cycle (indicating large amounts of reversible martensitic transformation) later showed high residual strain at the end of cycling. A possible explanation for this is that $\mathrm{CV}$ formation and recovery is not as favorable a mechanism as HPV formation and recovery.

- Linear combinations of HPVs, which lie on the same planes in strain space as CV/HPV combinations, showed no elevated residual strain accumulation compared to single HPV solutions.

- The saturation of residual strain and stabilization of $\mathrm{CV}$ fraction that occurred with cycling corresponded to macroscopic shakedown and led to a more uniform distribution of HPVs. Shakedown can be attributed in part to a shift in martensite volume fractions from 
configurations that are incompatible with the parent austenite to compatible configurations that are stabilized by plastic deformation.

- The location of stable (shaken down) martensite depended on the amount of reorientation present in the local microstructure. When transformation history was not taken into account, a high volume fraction of martensite continued to transform in HPV configurations with cycling. However, when transformation was limited to configurations that appeared in cycle one, the stable transforming martensite arrangement was a much lower volume fraction composition that included remnant $\mathrm{CV}$.

- Similarly, the degree of reorientability of martensite during cyclic superelastic transformation informed the source of residual strain. Analysis using the history-free assumption of infinitely reorientable martensite resulted in a stable, shaken-down transformation configuration with a high volume fraction of martensite that necessitated the presence of primarily plastic deformation in the residual strain field. In comparison, the strict-history case - using the assumption of no martensite reorientation - resulted in the retention of approximately $50 \%$ of the martensite associated with non-HPV solutions and $30 \%$ of pure HPV configurations as residual strain.

- Future work to determine the amount of residual strain attributable to martensitic transformation and the degree of reorientation during superelastic cycling requires the development of new experimental capabilities. Tracking reorientation by diffraction-based methods could enable the determination of the location of shaken-down martensite between the infinitely reorientable and strictly history dependent solutions.

\section{$\underline{\text { VI. Acknowledgements }}$}

This work was supported by the U.S. Department of Energy, Office of Science, Basic Energy Sciences, under Award \#DE-SC0003996. The authors thank Prof. John Shaw for helpful discussions and valuable insight as well as Dr. Adam Kammers for his assistance with nanoparticle patterning. Portions of this work were performed using the FEI Quanta Dual Beam FIB at the Electron Microbeam Analysis Lab at the University of Michigan. 


\section{Figures}
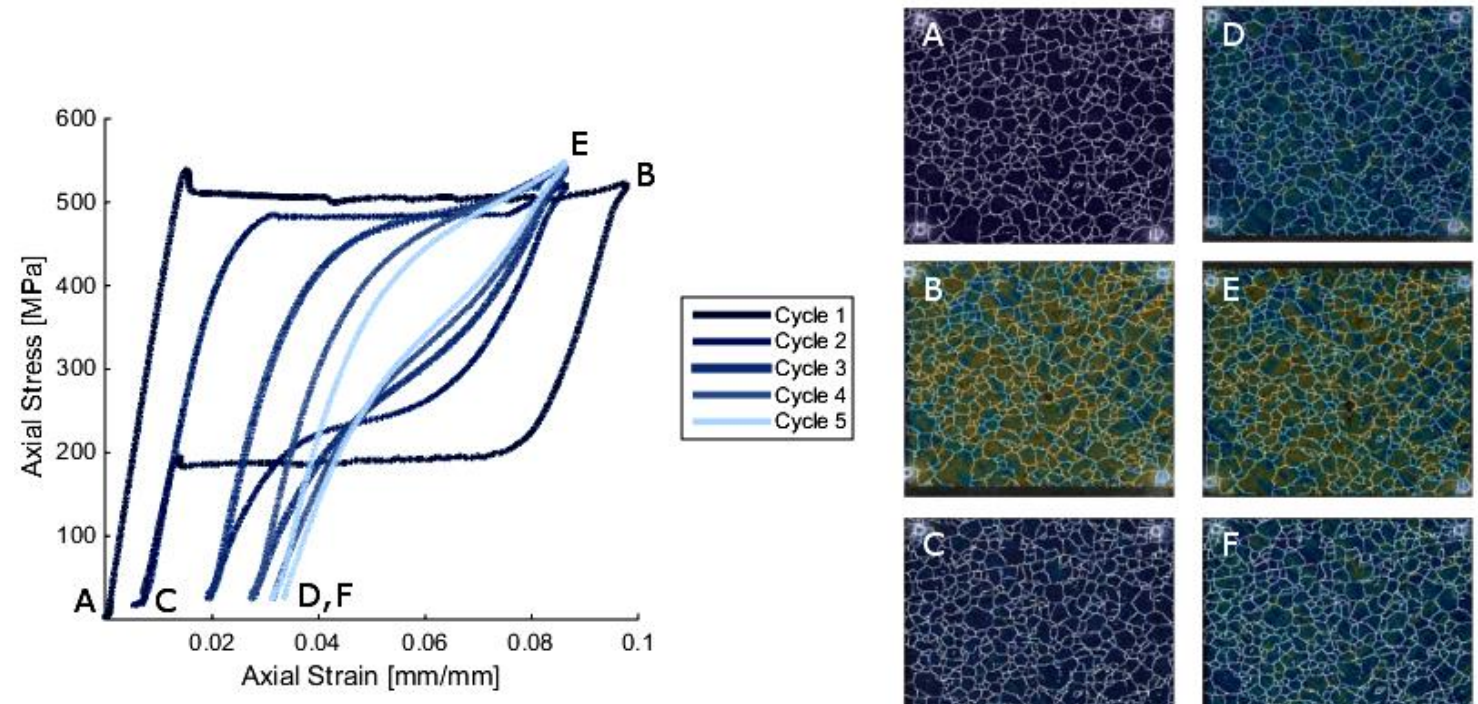

Axial
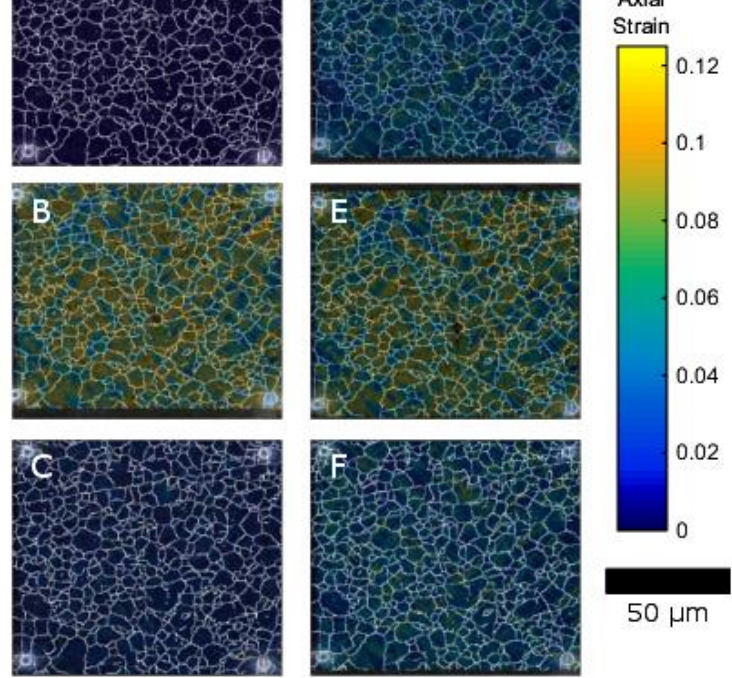

Figure 1: (Left image) Evolution of the macroscopic stress-strain response with cycling, and (right image) the associated strain fields of a $100 \mu \mathrm{m} \times 100 \mu \mathrm{m}$ area of interest for the first $(\mathrm{ABC})$ and fifth (DEF) superelastic cycle. The material is considered 'fully transformed' at the end of the superelastic stress plateau (Point B), where the stress starts to rise above the initial transformation stress. Similar points where the local strain matches the first cycle 'full' transformation (Point E) can be defined for each subsequent cycle. In the local strain maps, grain boundaries as determined by EBSD are highlighted in white. For color, please see the online pdf version. 


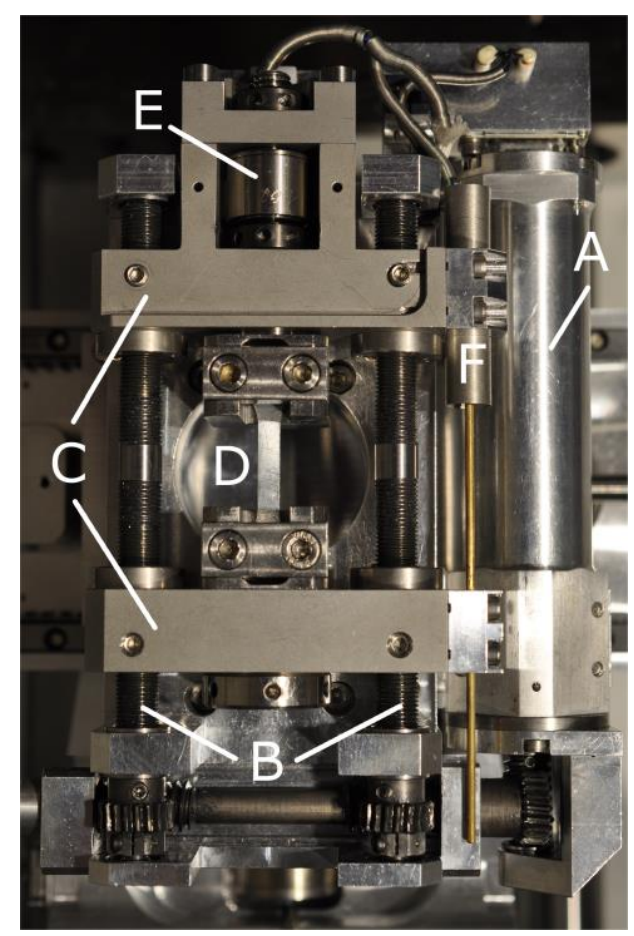

Figure 2: Kammrath and Weiss Load Frame used for in situ experiments. Marked items are A: High torque stepper motor, B: Counter-rotating screw drives, C: Equal displacement crossheads to keep the sample centered during testing, D: Alignment sample in grips, E: Interchangeable load cell ( $1 \mathrm{kN}$ capacity used for testing), F: LVDT Linear Encoder for secondary macroscopic strain measurements. 
All Martensite Configurations in Grain 9618

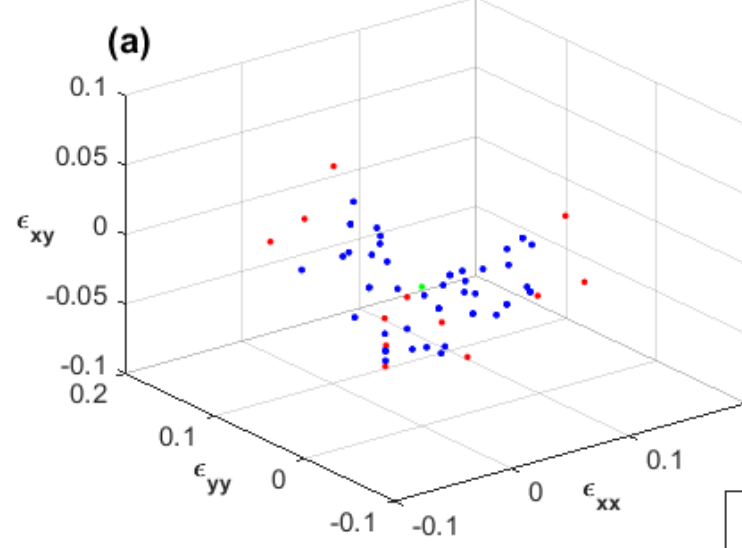

Fit to Two Compatible HPVs

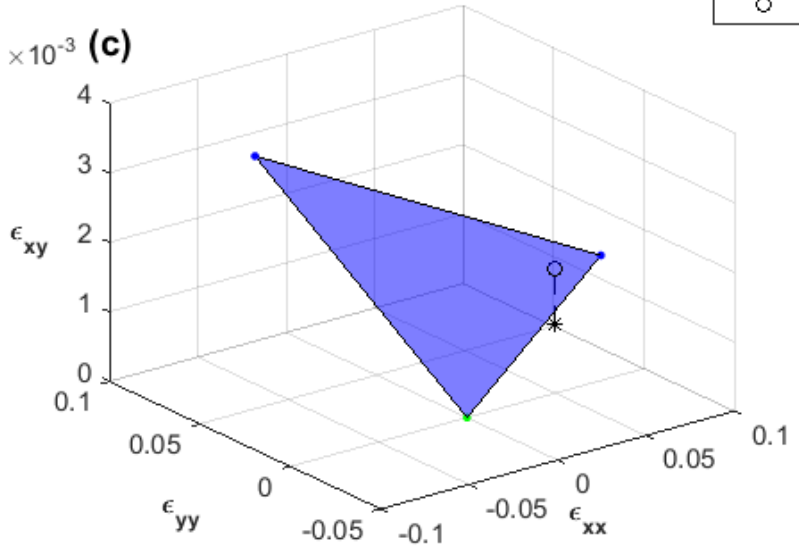

Fit to Single HPV
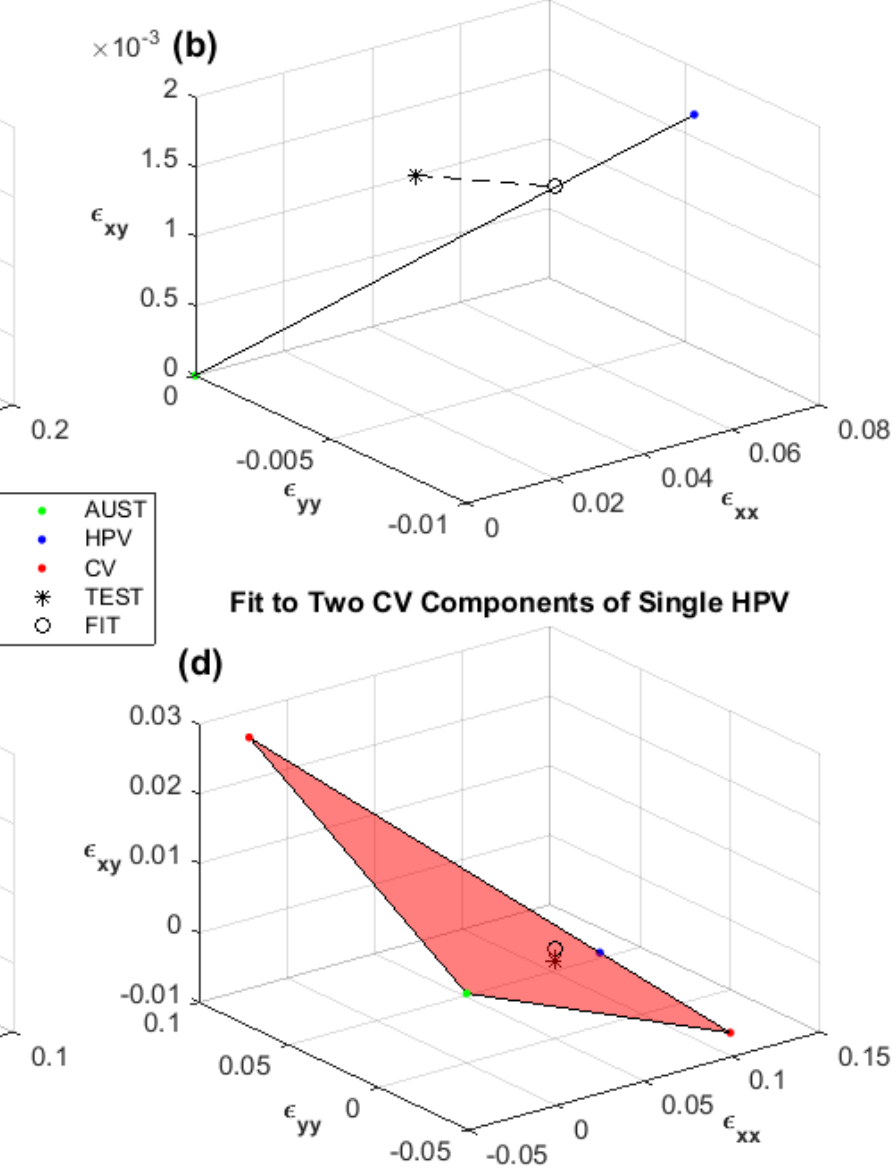

Figure 3: (a) Distribution of CV and HPV strain points in an individual grain (Grain \#9618). (b) A graphical representation of fitting a test point $\left(^{*}\right)$ to the best match phase mixture of a single HPV and austenite. Similar representations of (c) fitting the test point to a three-phase mixture can be constructed for multiple HPVs and (d) the decomposition of a single HPV into its CV components. 


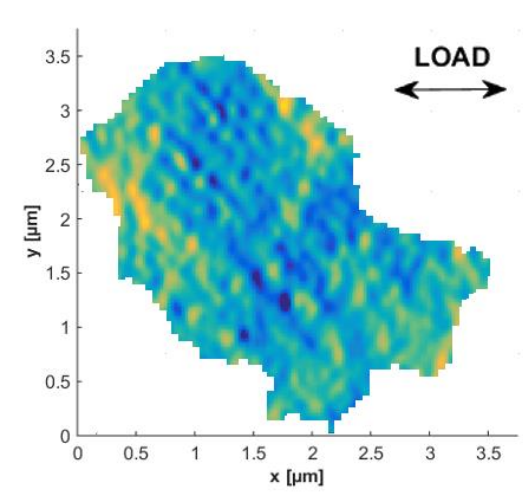

(a)

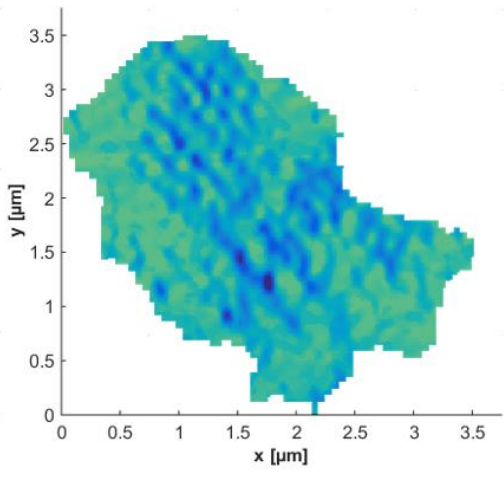

(b)

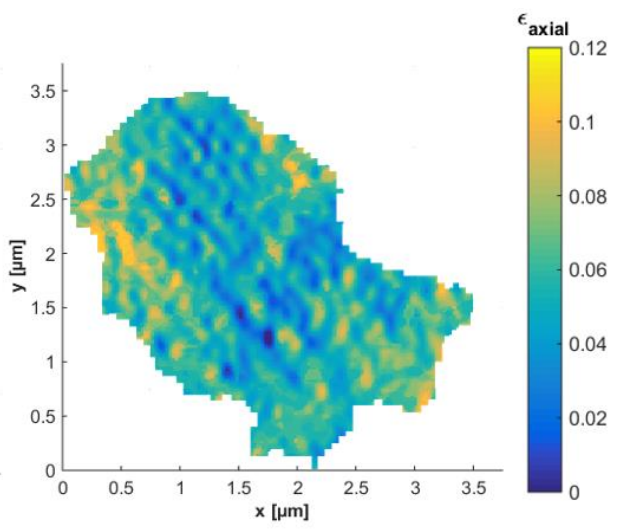

(c)

Figure 4: (a) Recovered axial strain measured during the first cycle of superelastic deformation in a single grain. (b) The best match of axial strain using only the HPV solutions available for the grain orientation. (c) The best match of axial strain using both the available HPV and CV solution sets. The vast improvement of the model fit after including the CV solutions is taken as strong evidence of reorientation of HPVs to their component CVs within the first superelastic cycle.

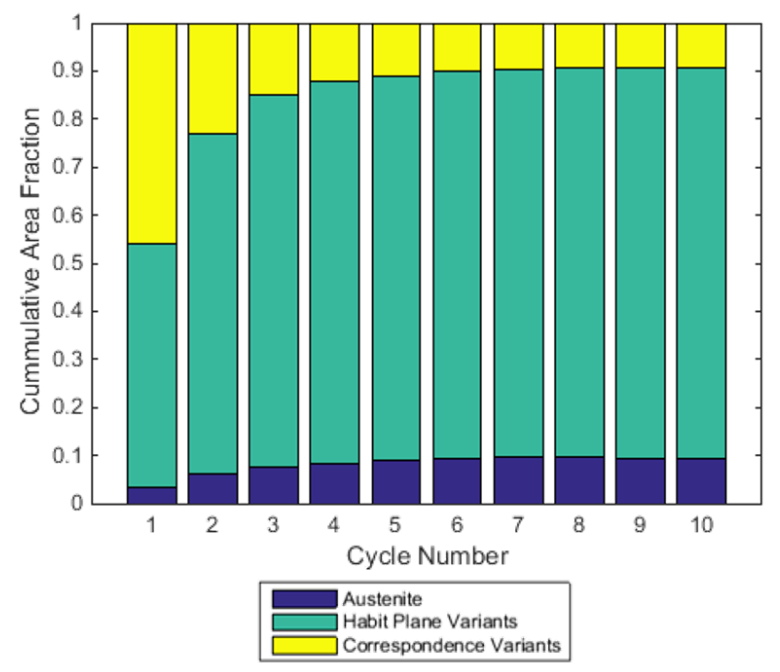

Figure 5: Distribution of phase fraction in the recovered strain fit for cycles 1-10. Note the stabilization of phase fraction around cycle 5 or 6 that correlates with the end of major shakedown of the macroscopic stress-strain response. 

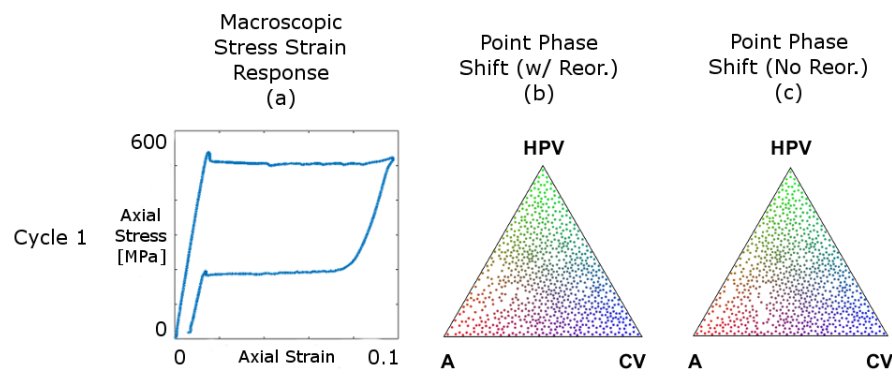

Residual Strain

(c)

Accumulation
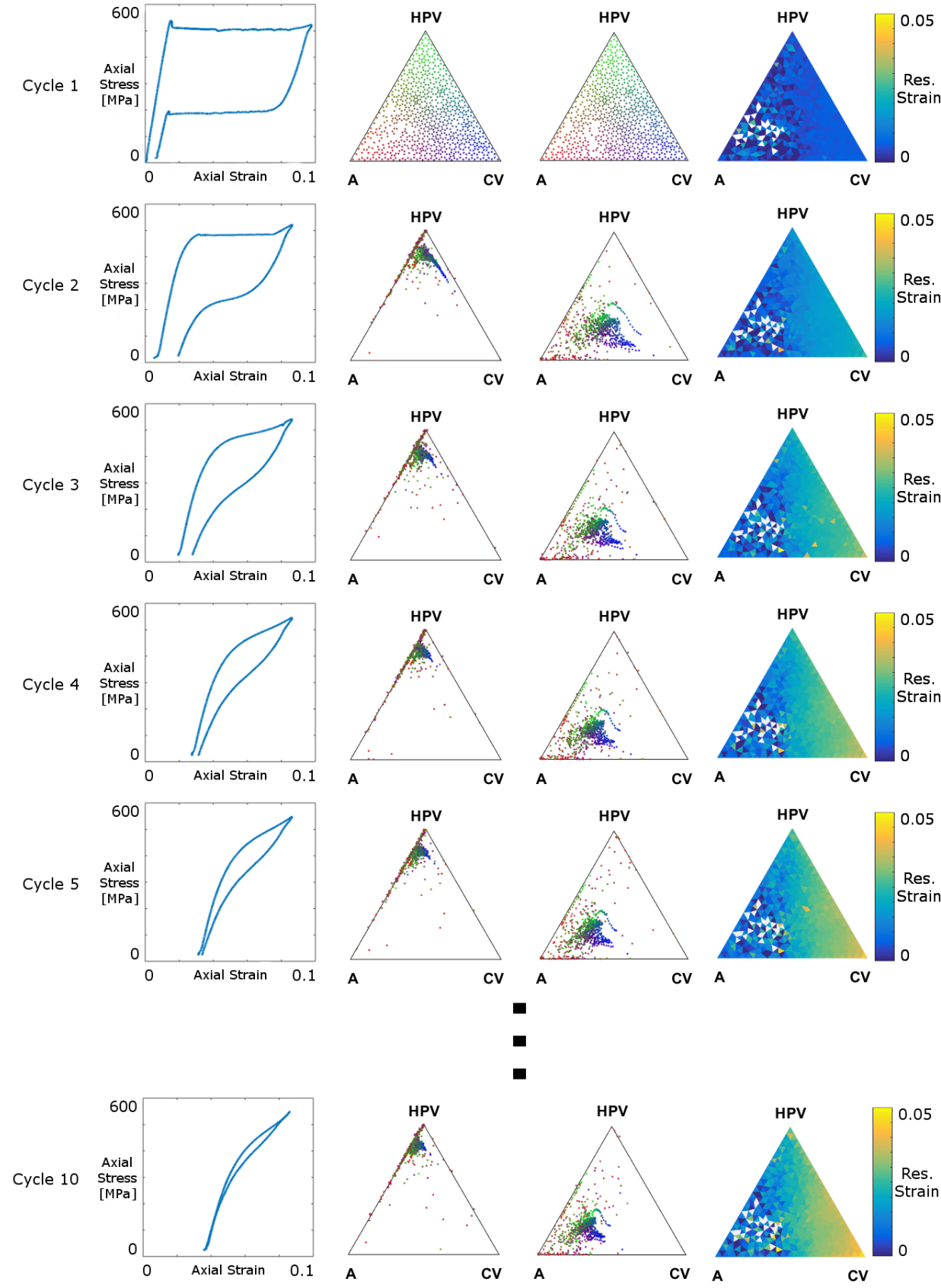

Figure 6: (a) The evolution of the macroscopic stress-strain response with superelastic cycling. (b/c) Tracking points with particular volume fractions in their initial recoverable transformation as they collapse to a more uniform HPV set with cycling. (d) The mean residual strain accumulated in the material based on the initial transformation volume fractions. Note the heightened accumulation of residual strain in material that initially transforms to high $\mathrm{CV}$ fractions. 


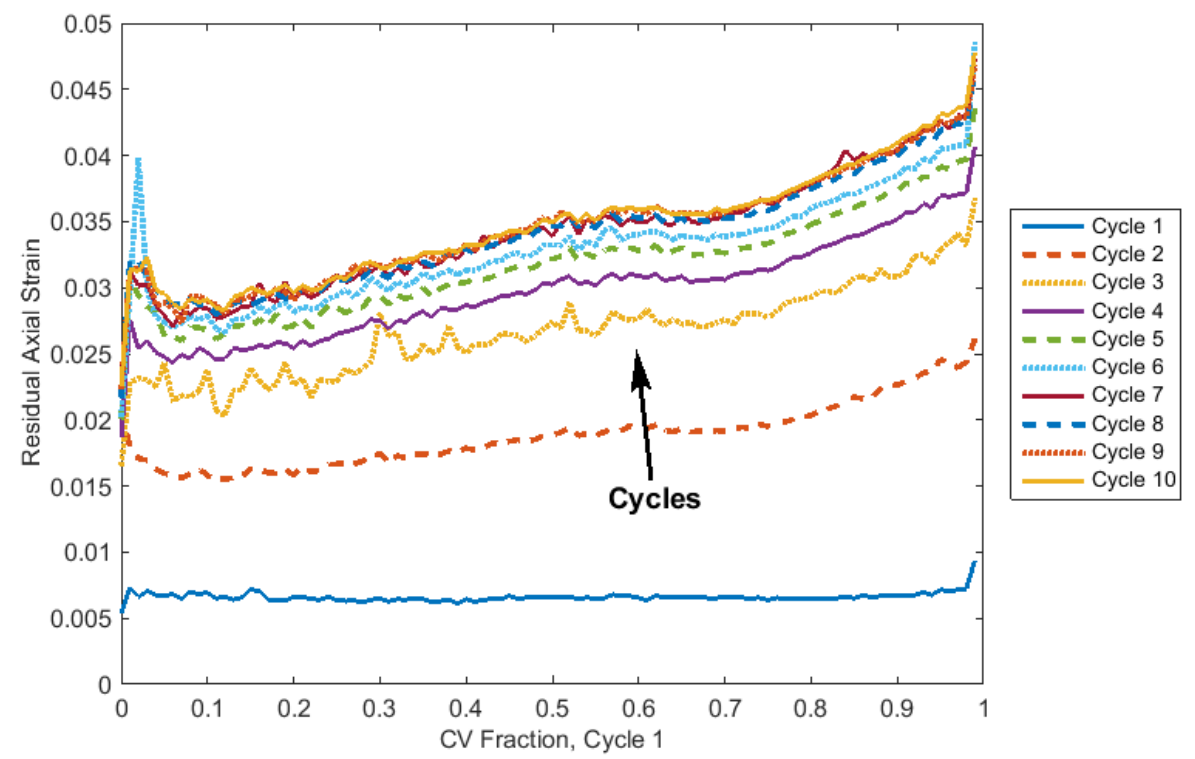

Figure 7: Mean residual strain accumulation in the sample over ten superelastic cycles as a function of the $\mathrm{CV}$ fraction of Cycle 1. Residual strain accumulates fairly linearly with increasing $\mathrm{CV}$ fraction in the initial martensite transformation configuration.

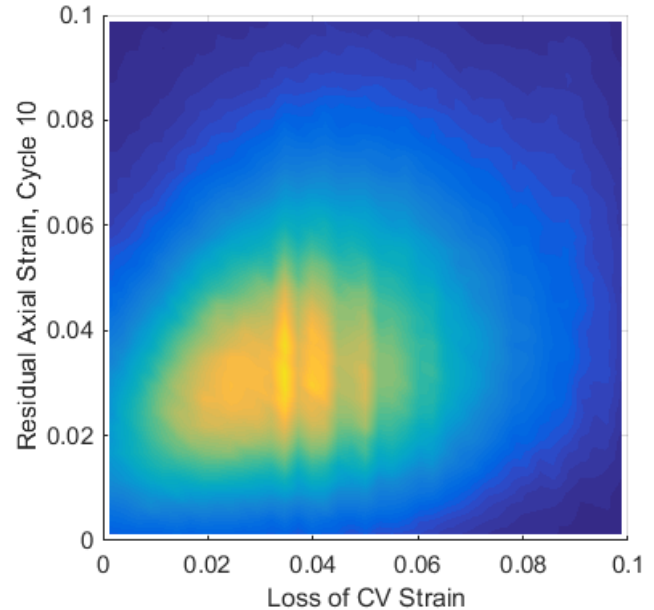

(a)

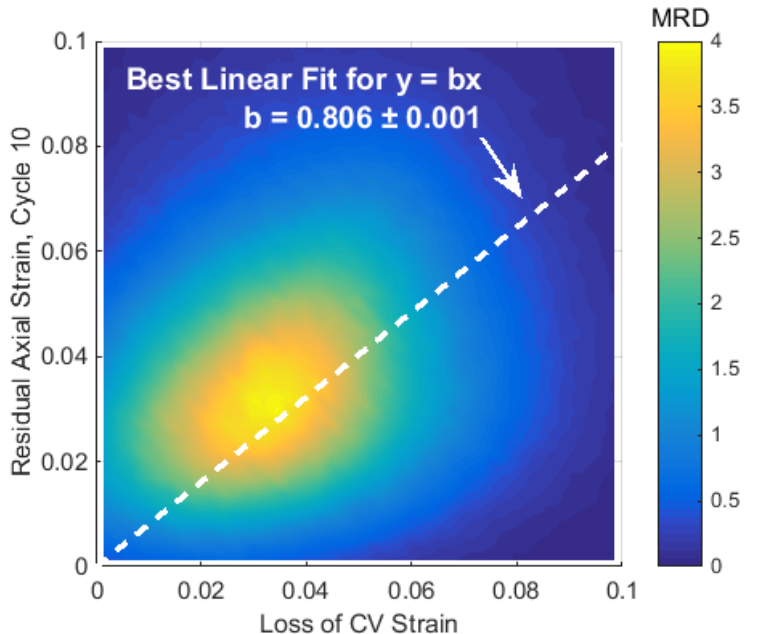

(b)

Figure 8: Relationship between loss of strain from correspondence variants using the (a) historyfree assumption, where there is no penalty for reorientation and transformation history is not considered; and using the (b) strict history assumption, where the previous transformation configuration fixes the martensite configurations available in subsequent cycles. 


\section{$\underline{\text { References }}$}

Ball, J., James, R.D., 1989. Fine phase mixtures as minimizers of energy. Anal. Contin. Mech.

Bhattacharya, K., 2003. Microstructure of Martensite: Why it forms and how it gives rise to the shape memory effect. Oxford University Press Inc., New York.

Bhattacharya, K., Kohn, R.V., 1997. Elastic Energy Minimization and the Recoverable Strains of Polycrystalline Shape-Memory Materials. Arch. Ration. Mech. Anal. 139, 99-180. doi:10.1007/s002050050049

Bhattacharya, K., Kohn, R.V., 1996. Symmetry, texture and the recoverable strain of shapememory polycrystals. Acta Mater. 44, 529-542. doi:10.1016/1359-6454(95)00198-0

Bowers, M.L., Chen, X., De Graef, M., Anderson, P.M., Mills, M.J., 2014. Characterization and modeling of defects generated in pseudoelastically deformed NiTi microcrystals. Scr. Mater. 78-79, 69-72. doi:10.1016/j.scriptamat.2014.02.001

Chen, X., Srivastava, V., Dabade, V., James, R.D., 2013. Study of the cofactor conditions: Conditions of supercompatibility between phases. J. Mech. Phys. Solids 61, 2566-2587. doi:10.1016/j.jmps.2013.08.004

Delville, R., Kasinathan, S., Zhang, Z., Humbeeck, J. Van, James, R.D., Schryvers, D., 2010. Transmission electron microscopy study of phase compatibility in low hysteresis shape memory alloys. Philos. Mag. 90, 177-195. doi:10.1080/14786430903074755

DesRoches, R., McCormick, J., Delemont, M., 2004. Cyclic Properties of Superelastic Shape Memory Alloy Wires and Bars. J. Struct. Eng. 130, 38-46. doi:10.1061/(ASCE)07339445(2004)130:1(38)

Eggeler, G., Hornbogen, E., Yawny, A., Heckmann, A., Wagner, M.F.-X., 2004. Structural and functional fatigue of NiTi shape memory alloys. Mater. Sci. Eng. A 378, 24-33. doi:10.1016/j.msea.2003.10.327

Frick, C.P., Clark, B.G., Schneider, A.S., Maaß, R., Petegem, S. Van, Swygenhoven, H. Van, 2010. On the plasticity of small-scale nickel-titanium shape memory alloys. Scr. Mater. 62, 492-495. doi:10.1016/j.scriptamat.2009.12.023 
Gall, K., Sehitoglu, H., 1999. The role of texture in tension-compression asymmetry in polycrystalline NiTi. Int. J. Plast. 15, 69-92.

Hane, K., Shield, T.W., 1999. Microstructure in the cubic to monoclinic transition in titaniumnickel shape memory alloys. Acta Mater. 47, 2603-2617. doi:10.1016/S13596454(99)00143-3

Kammers, A.D., Daly, S.H., 2013a. Self-assembled nanoparticle surface patterning for improved digital image correlation in a scanning electron microscope. Exp. Mech. 53, 1333-1341. doi:10.1007/s11340-013-9734-5

Kammers, A.D., Daly, S.H., 2013b. Digital image correlation under scanning electron microscopy: Methodology and validation. Exp. Mech. 53, 1743-1761. doi:10.1007/s11340013-9782-x

Kang, G., 2013. Advances in transformation ratcheting and ratcheting-fatigue interaction of NiTi shape memory alloy. Acta Mech. Solida Sin. 26, 221-236. doi:10.1016/S08949166(13)60021-X

Kang, G., Kan, Q., Qian, L., Liu, Y., 2009. Ratchetting deformation of super-elastic and shapememory NiTi alloys. Mech. Mater. 41, 139-153. doi:10.1016/j.mechmat.2008.09.001

Kim, K., Daly, S.H., 2013. The effect of texture on stress-induced martensite formation in nickel-titanium. Smart Mater. Struct. 22, 075012. doi:10.1088/0964-1726/22/7/075012

Kim, K., Daly, S.H., 2010. Martensite Strain Memory in the Shape Memory Alloy NickelTitanium Under Mechanical Cycling. Exp. Mech. 51, 641-652. doi:10.1007/s11340-0109435-2

Kimiecik, M.G., Jones, J.W., Daly, S.H., 2013. Quantitative Analysis of Phase Transformation in Ni-Ti Shape Memory Alloys. Adv. Mater. Process. 21, 21-25.

Knowles, K., Smith, D., 1980. The crystallography of the martensitic transformation in equiatomic Nickel-Titanium. Acta Metall. 29, 101-110.

Kumar, P.K., Caer, C., Atkinson, G., Patoor, E., Lagoudas, D.C., 2011. The influence of stress and temperature on the residual strain generated during pseudoelastic cycling of NiTi SMA wires 7978, 79781E-79781E-9. doi:10.1117/12.881994 
Madangopal, K., 1997. The self accommodating martensitic microstructure of NiTi shape memory alloys. Acta Mater. 45, 5347-5365.

Matsumoto, O., Miyazaki, S., Otsuka, K., Tamura, H., 1987. Crystallography of martensitic transformation in Ti-Ni single crystals. Acta Metall. 35, 2137-2144. doi:10.1016/00016160(87)90042-3

Miyazaki, S., Kimura, S., Otsuka, K., Suzuki, T., 1984. The habit plane and transformation strains associated with the martensitic transformation in Ti-Ni single crystals. Scr. Metall. $18,883-888$.

Miyazaki, S., Kimura, S., Takei, F., Miura, T., Otsuka, K., Suzuki, T., 1983. Shape memory effect and pseudoelasticity in a Ti-Ni single crystal. Scr. Metall. 17, 1057-1062. doi:10.1016/0036-9748(83)90453-2

Miyazaki, S., No, V., Kitamura, K., Khantachawana, A., Hosoda, H., 2000. Texture of Ti-Ni rolled thin plates and sputter-deposited thin films. Int. J. Plast. 16, 4-8.

Miyazaki, S., Otsuka, K., Wayman, C.M., 1989a. The shape memory mechanism associated with the martensitic transformation in Ti-Ni alloys-I. Self-accommodation. Acta Metall. 37, 1873-1884. doi:10.1016/0001-6160(89)90072-2

Miyazaki, S., Otsuka, K., Wayman, C.M., 1989b. The shape memory mechanism associated with the martensitic transformation in Ti-Ni alloys-II. Variant coalescence and shape recovery. Acta Metall. 37, 1885-1890.

Norfleet, D., Sarosi, P., Manchiraju, S., Wagner, M.F.-X., Uchic, M.D., Anderson, P.M., Mills, M.J., 2009. Transformation-induced plasticity during pseudoelastic deformation in Ni-Ti microcrystals. Acta Mater. 57, 3549-3561. doi:10.1016/j.actamat.2009.04.009

Ortin, J., Planes, A., Delaey, L., 2006. Hysteresis in shape-memory materials. Sci. Hysteresis 3, 467-553. doi:10.1016/B978-012480874-4/50023-3

Pelton, A.R., 2011. Nitinol Fatigue: A Review of Microstructures and Mechanisms. J. Mater. Eng. Perform. 20, 613-617. doi:10.1007/s11665-011-9864-9

Pelton, A.R., Schroeder, V., Mitchell, M.R., Gong, X.-Y., Barney, M., Robertson, S.W., 2008. Fatigue and durability of Nitinol stents. J. Mech. Behav. Biomed. Mater. 1, 153-64. doi:10.1016/j.jmbbm.2007.08.001 
Pfetzing-Micklich, J., Ghisleni, R., Simon, T., Somsen, C., Michler, J., Eggeler, G., 2012.

Orientation dependence of stress-induced phase transformation and dislocation plasticity in NiTi shape memory alloys on the micro scale. Mater. Sci. Eng. A 538, 265-271. doi:10.1016/j.msea.2012.01.042

Rao, A., Ruimi, A., Srinivasa, A.R., 2014. Internal loops in superelastic shape memory alloy wires under torsion - Experiments and simulations/predictions. Int. J. Solids Struct. 51, 4554-4571. doi:10.1016/j.ijsolstr.2014.09.002

Reu, P.L., 2013. Uncertainty Quantification for 3D Digital Image Correlation.

Richards, A., Lebensohn, R., Bhattacharya, K., 2013. Interplay of martensitic phase transformation and plastic slip in polycrystals. Acta Mater. 61, 4384-4397.

Robertson, S.W., Pelton, A.R., Ritchie, R.O., 2011. Mechanical fatigue and fracture of Nitinol. Int. Mater. Rev. 57, 1-36. doi:10.1179/1743280411Y.0000000009

Schreier, H.W., Orteu, J.-J., Sutton, M.A., 2009. Image Correlation for Shape, Motion and Deformation Measurements. Springer US, Boston, MA. doi:10.1007/978-0-387-78747-3

Shaw, J.A., 1997. Material Instabilites in a Nickel-Titanium Shape Memory Alloy. University of Texas.

Shi, H., Delville, R., Srivastava, V., James, R.D., Schryvers, D., 2014. Microstructural dependence on middle eigenvalue in Ti-Ni-Au. J. Alloys Compd. 582, 703-707. doi:10.1016/j.jallcom.2013.08.132

Song, Y., Chen, X., Dabade, V., Shield, T.W., James, R.D., 2013. Enhanced reversibility and unusual microstructure of a phase-transforming material. Nature 502, 85-88. doi:10.1038/nature 12532

Stebner, A., Vogel, S.C., Noebe, R.D., Sisneros, T.A., Clausen, B., Brown, D.W., Garg, A., Brinson, L.C., 2013. Micromechanical quantification of elastic, twinning, and slip strain partitioning exhibited by polycrystalline, monoclinic nickel-titanium during large uniaxial deformations measured via in-situ neutron diffraction. J. Mech. Phys. Solids 61, 23022330. doi:10.1016/j.jmps.2013.05.008

Sutton, M.A., Li, N., Garcia, D., Cornille, N., Orteu, J.-J., McNeill, S.R., Schreier, H.W., Li, X., Reynolds, A.P., 2007a. Scanning electron microscopy for quantitative small and large 
deformation measurements Part II: Experimental validation for magnifications from 200 to 10,000. Exp. Mech. 47, 789-804. doi:10.1007/s11340-007-9041-0

Sutton, M.A., Li, N., Joy, D.C., Reynolds, A.P., Li, X., 2007b. Scanning electron microscopy for quantitative small and large deformation measurements Part I: SEM imaging at magnifications from 200 to 10,000. Exp. Mech. 47, 775-787. doi:10.1007/s11340-0079042-z

Wang, Y.Q., Sutton, M.A., Ke, X.-D., Schreier, H.W., Reu, P.L., Miller, T.J., 2011. On Error Assessment in Stereo-based Deformation Measurements. Exp. Mech. 51, 405-422. doi:10.1007/s11340-010-9449-9

Yamamoto, H., Taya, M., Liang, Y., Namli, O.C., Saito, M., 2013. Fatigue properties of NiTi shape-memory alloy thin plates 8689, 86890S. doi:10.1117/12.2009857 